\title{
Stationary Modes and Localized Metastable States in a Triangular Lattice of Active Particles
}

\author{
K.S.Sergeev, S. V. Dmitriev, E. A. Korznikova, A.P. Chetverikov
}

The dynamics of a triangular lattice consisting of active particles is studied. Particles with nonlinear friction interact via nonlinear forces of Morse potential. Nonlinear friction slows down fast particles and accelerates slow ones. Each particle interacts mainly with the nearest neighbors due to the choice of the cut-off radius.

Stationary modes (attractors) and metastable states of the lattice are studied by methods of numerical simulation.

It is shown that the main attractor of the system under consideration is the so-called translational mode - the state with equal and unidirectional velocities of all particles. For some parameter values translational modes with defects in the form of vacancies and interstitial particles are possible.

Metastable localized states are presented by the plane soliton-like waves ( $M$-solitons) with inherent velocity and density maxima. The lifetime of such states depends on the lattice parameters and the wavefront width. All metastable states transform into the translational mode after a transient process.

Keywords: lattices, active particles, solitons, Morse potential

Received April 06, 2018

Accepted June 01, 2018

This work was supported by the Russian Science Foundation, grant no. 16-12-10175.

\author{
Konstantin S. Sergeev \\ kssergeev@mail.ru \\ Alexander P. Chetverikov \\ chetverikovap@info.sgu.ru \\ National Research Saratov State University \\ ul. Astrakhanskaya 83, Saratov, 410012 Russia \\ Sergey V. Dmitriev \\ dmitriev . sergey . v@gmail.com \\ Institute for Metals Superplasticity Problems RAS \\ ul. Khalturina 39, Ufa, 450001 Russia \\ National Research Tomsk State University \\ ul. Lenina 36, Tomsk, 634050 Russia \\ Elena A. Korznikova \\ elena.a.korznikova@gmail.com \\ Institute for Metals Superplasticity Problems RAS \\ ul. Khalturina 39, Ufa, 450001 Russia
}

RUSSIAN JOURNAL OF NONLINEAR DYNAMICS, 2018, 14(2), 195-207 


\section{Introduction}

A wide class of systems able to extract energy from its environment and transform it to kinetic energy is called active materials (active matter). Dissipation and energy pumping take place at a microscopic level, but interacting particles exhibit collective (macroscopic) dynamics. There exist a number of different models of active systems with different mechanisms of energy conversion and particles interaction (see, e.g., [1]). They demonstrate novel dynamical features which are not observed in conservative systems. An example of such behavior is pattern formation $[2-5]$. The results of those studies suggest that it is possible to create active materials with different novel features.

By now, synthetic prototypes of active materials have been created: the so-called "active colloids" in the form of micron-sized spheres partially coated by a catalyst. This catalyst causes a chemical reaction with environment component and, as a result, particles get a momentum and begin to move [6].

Suspensions of conservative and active solid particles ("swimmers") are also actively investigated $[7,8]$. Models of active particle ensembles interacting via common velocity field are used to investigate swimmers' dynamics [1, 9-11].

In nonlinear optics, a great deal of attention is paid to open PT-symmetric systems with a balanced gain and loss of energy $[12,13]$. PT-symmetric Klein-Gordon models with viscous friction, where areas with positive and negative friction coefficient alternate, are also very interesting [14-18].

Investigations of the properties of active polymers and membranes [5, 19-22] stimulate the development of models of two-dimensional ensembles and lattices of active particles interacting via potential forces.

A hard-core interaction of finite-size particles is usually softened in the models to avoid discontinuities and singularities when particles overlap. Therefore, in models the particles are often considered to be pointwise, and nonlinear potentials with relatively soft interactions, for example, the truncated and displaced Lennard - Jones potential [23] or the Morse potential [24, 25], are usually used to describe the interparticle bonds.

In this paper we consider the dynamics of one of the examples of active matter - a lattice of active particles with potential interaction. There exists a large body of literature on different localized excitations in conservative lattices (see, e.g., [25-35]). In those works it has been found that solitons and mobile discrete breathers of different configurations can propagate in two-dimensional lattices.

For a one-dimensional chain of active particles with nonlinear friction, interacting via potential forces [36-39], it was shown that stationary modes (attractors) of such systems with periodic boundary conditions are cnoidal waves with uniform spatial distribution of density maxima.

When the width of density peaks is significantly smaller than the distance between them, these modes can be considered as an ordered ensemble of dissipative discrete solitons [40]. It is also shown that the processes of establishing the stationary modes can be very long.

In this paper we present the results of a study of stationary modes and metastable states observed in a two-dimensional lattice of active particles interacting via Morse potential forces. The main types of stationary modes and various localized metastable modes of a system with periodic boundary conditions are described. The conditions for their excitation are indicated and the lifetimes are estimated.

This paper is organized as follows. In Section 2 we introduce a mathematical model of the system of interest and explain the main features of the model. In Section 3 we discuss 
stationary modes which are attractors of the dynamical system considered. Section 4 is devoted to the description of discovered metastable regimes, determination of their lifetimes with different parameter values and initial conditions. Also in this part we apply the dynamical structure factor as an instrument for identification of some modes. Finally, the last section contains our conclusions and a summary of all detected dynamical regimes.

\section{Model and equations}

We consider a triangular lattice with periodic boundary conditions consisting of $10^{3}$ active pointwise particles. The particles experience negative friction with the nonlinear velocitydependent coefficient

$$
\gamma\left(\vec{v}_{i}\right)=\widetilde{\mu}\left(1-\frac{\left|\vec{v}_{i}\right|^{2}}{\widetilde{v}_{0}^{2}}\right),
$$

where $\widetilde{\mu}$ is the linear coefficient of negative friction, $\vec{v}_{i}$ is velocity of particle $i$ and $\widetilde{v}_{0}$ is the absolute value of stationary velocity. The velocity of each particle tends to reach the velocity $\left|v_{i}\right|=\widetilde{v}_{0}$. Note that the nonlinear Rayleigh friction under consideration is not sensitive to the velocity direction, but only to its absolute value.

The interaction between particles is determined by the modified Morse potential [25]

$$
U(z)=D\left(e^{-2 b(z-\sigma)}-2 e^{-b(z-\sigma)}\right) \frac{1}{1+e^{(z-d) / 2 \nu}},
$$

where $z$ is the distance between particles, $D$ is the potential well depth, $b$ is the rigidity of the potential, $\sigma$ is the equilibrium distance between particles, and $d$ and $\nu$ are the parameters of the modified potential.

Parameters $d$ and $\nu$ are chosen so that the potential and its derivative are close to zero at distances greater than the cut-off radius $R$ (Fig. 1a). The particles interact only if the distances between them are less than $R$. In this paper, we study the lattices with nearest-neighbor interactions.

The size of the simulation cell and the initial positions of the particles are chosen in such a way that a triangular lattice is formed (Fig. 1b). The parameters of the potential $b \sigma=5$, $d=1.35 \sigma$ and $\nu=0.035 \sigma$ correspond to the cut-off radius $R=1.6 \sigma$, and mainly the neighboring particles interact.

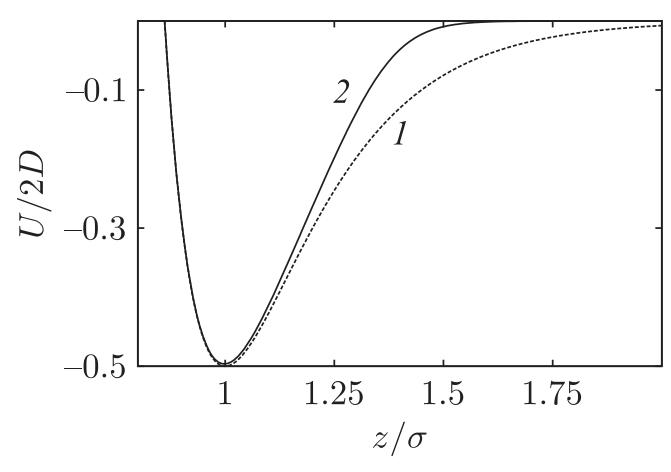

(a)

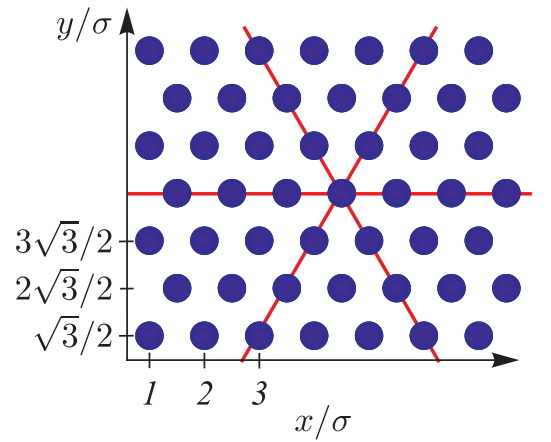

(b)

Fig. 1. (a) Profiles of the traditional (line 1) and modified (line 2) Morse potentials with parameter values $b \sigma=5, d=1.35 \sigma$ and $\nu=0.035 \sigma$. (b) Schematic representation of a lattice with triangular symmetry; lines show the crystallographic axes. 
The dynamics of the $i$-th particle is governed by the equations of motion in the dimensionless variables:

$$
\begin{aligned}
& \ddot{\vec{q}}_{i}-\mu\left(1-\frac{\left|\dot{\vec{q}}_{i}^{2}\right|}{v_{0}^{2}}\right) \dot{\vec{q}}_{i}= \\
& =\sum_{\left|\vec{q}_{i}^{k}\right|<R} \frac{\vec{q}_{i}^{k}}{\left|\vec{q}_{i}^{k}\right|}\left[\left(e^{\left(b \sigma-\left|\vec{q}_{i}^{k}\right|\right)}-e^{2\left(b \sigma-\left|\vec{q}_{i}^{k}\right|\right)}\right) \cdot \frac{1}{1+e^{\frac{\left|\vec{q}_{i}^{k}\right| / b-d}{2 \nu}}}-\frac{1}{2 b} \frac{e^{2\left(b \sigma-\left|\vec{q}_{i}^{k}\right|\right)}-2 e^{\left(b \sigma-\left|\vec{q}_{i}^{k}\right|\right)}}{\left.2 \nu e^{\frac{\left|\vec{q}_{i}^{k}\right| / b-d}{2 \nu}}\right] .}\right.
\end{aligned}
$$

Here we use the dimensionless coordinate $\vec{q}_{i}=b \vec{r}_{i}$ (where $\vec{r}_{i}=\left\{x_{i} ; y_{i}\right\}$ define the actual coordinates of particles), $\dot{\vec{q}}_{i}=\frac{\omega_{c}}{b} \vec{v}_{i}$ is the dimensionless velocity, where the overdot means differentiation with respect to the dimensionless time $\tau=\omega_{c} t$. The parameter $\mu=\widetilde{\mu} \omega_{c} / b$ is a dimensionless linear negative friction coefficient, $v_{0}=\widetilde{v}_{0} \frac{b}{\omega_{M}}$ is the stationary velocity value, $\left|\vec{q}_{i}^{k}\right|$ is the dimensionless distance between particles $i$ and $k$ and $\frac{\vec{q}_{i}^{k}}{\left|\vec{q}_{i}^{k}\right|}$ is the unit vector directed from the $i$ th particle to the $k$ th one.

Thus, in our notations the unit of time is $1 / \omega_{c}$, and the unit of energy is $2 D$. Here $\omega_{c}=\sqrt{2 D b^{2} / m}$ is a frequency of small oscillations in a Morse potential well where $m$ is the mass of a particle. But further in the text we will present the velocity in the units of $\omega_{c} \sigma$, which is a "sound velocity" in the corresponding 1D Morse chain with the same values of parameters [39].

In order to visualize the lattice dynamics, we will plot the particles by circles centered at the particle coordinates and Gaussian radial density distribution [25]

$$
\rho(\vec{r}, t)=\sum_{\left|\vec{r}-\vec{r}_{i}\right|<R} \exp \left(-\frac{\left(\vec{r}-\vec{r}_{i}\right)^{2}}{2 \lambda^{2}}\right),
$$

(see, e.g., Fig. 2a). This approach is very useful for visualization of different spatial structures.

\section{Stationary modes}

The system of interacting particles (2.3) has several characteristic values of energy, namely, the kinetic energy $m v_{0}^{2} / 2$, which corresponds to particle movement with stationary velocity $v_{0}$; the energy corresponding to the potential well depth $D$; and the energy of initially perturbed particle $m v_{r e l}^{2} / 2$ where $v_{r e l}$ is the particle velocity modulus relative to the moving simulation cell. Depending on the ratio of the characteristic values of energy, the dynamics of the ensemble can be different. In this work, the dynamics of the "lattice ensemble" is considered, in which the interactions between particles prevail on individual dynamics of particles. Therefore, the dynamics of such a lattice of active particles should reproduce certain features of the dynamics of the conservative lattice. However, the tendency of each particle to reach the stationary velocity value $v_{0}$ leads to new features of Morse lattice dynamics. Moreover, the evolution of excitations in such a lattice depends on the relation between the characteristic time scales of the system, such as the increment of linear perturbations, the time of nonlinear perturbations damping, and also on the Morse-oscillation period, which determines the characteristic scale of the collective 
motion. We analyze the behavior of the lattice with the fast increase of energy in the linear stage of evolution $(\mu \approx 1.4)$ and the short stage of nonlinear damping $\left(v_{0} \approx 0.14 \omega_{c} \sigma\right)$. The dynamics of the lattice with other types of behavior will be considered in forthcoming articles.

The main stationary state of the active lattice is the translational mode. In this state the entire lattice moves as a whole with the velocity $\vec{v}_{0}$, so that the velocities of all particles are equal and codirectional. In this trivial case the displacements from equilibrium positions are absent (Fig. 2a). This state is an attractor of the system considered: the most of metastable states transform to this translational mode. This mode restores kinetic and potential energies after a localized external impact, but the direction of the center of mass motion is not stable and can change under external influences.

In addition, there exists a large set of translational modes with defects in the lattice, as presented in Fig. 2b. The existence of such states is possible because the lattice can possess a local minimum of potential energy: the particle which is knocked out from its equilibrium position can get stuck between sites of the lattice. Such defects can arise, for example, as a result of propagation of a crowdion [30-32]. To excite the crowdion, one or several particles will be given initial momentum large enough to overcome the potential barrier and jump to the neighboring site [30].

In the model considered the crowdion excitation is possible, for example, if $\mu \lesssim 0.1$. Otherwise, the excited particles will be overdamped and lose their kinetic energy before the creation of a topological defect. Also, the crowdion excitation is possible when $v_{0}$ is large enough $\left(v_{0} \gtrsim 0.5\right)$; in this case the stationary value of the kinetic energy of the particles is comparable with the depth of the potential well. The dynamics of such lattices is a topic for a future study.

\section{Metastable states}

In addition to the stationary states-attractors (i.e., stationary modes) different metastable states can be excited by an appropriate choice of initial conditions. Metastable states are in fact transient processes from initial conditions to the stationary modes. Metastable states can

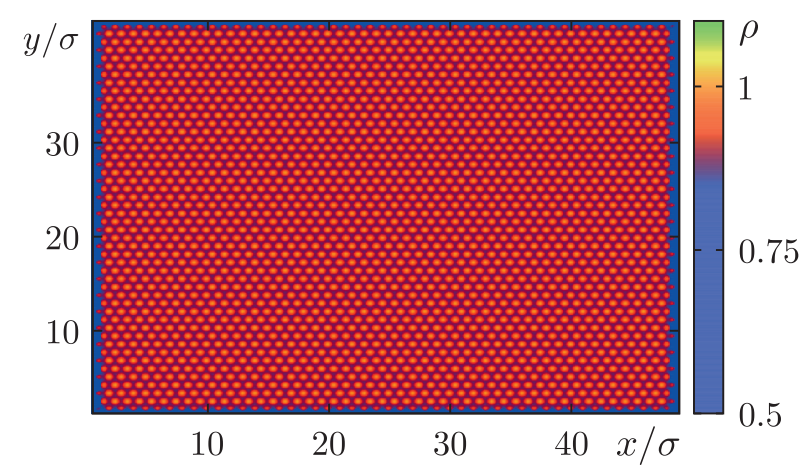

(a)

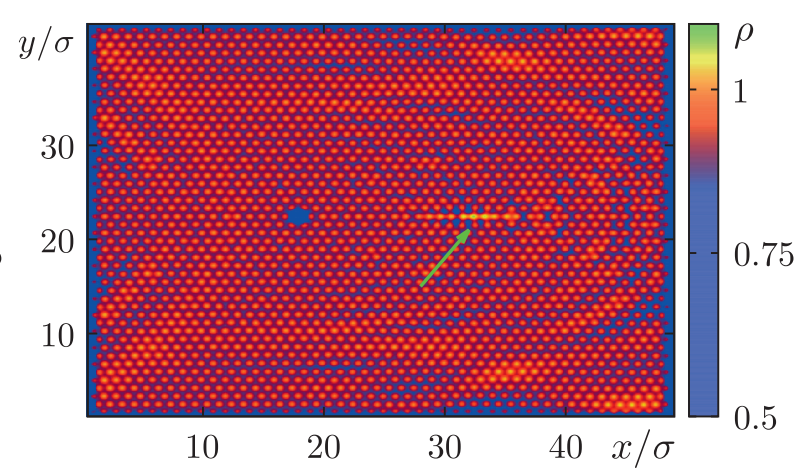

(b)

Fig. 2. Instantaneous spatial distributions of the particle density in the triangular lattice of $48 \times 48$ particles for the translational mode without defects at parameter values $b \sigma=5, \mu=1.41, v_{0}=0.14 \omega_{c} \sigma(\mathrm{a})$ and with defects in the form of a local compression of particles (shown by an arrow) induced by an interstitial particle knocked out from the site and a vacancy at the site from which this particle was knocked out. The parameter values are $b \sigma=4, \mu=0.07, v_{0}=0.14 \omega_{c} \sigma$ (b). 
be considered as quasi-stationary modes, which play an important role on the macroscopic characteristics of the system, because the duration of these processes can be very large. In real systems, which are under the influence of stochastic forces (i.e., at nonzero temperature) the disappearance of one of a metastable state is accompanied by the excitation of another. As a result, the system is in one of the metastable states all the time. For this reason, the determination of the features of metastable states and its lifetimes is an important question.

Results of numerical experiments show that a flat soliton-like wave (further denoted as "flat soliton") is the most long-living metastable state. It is the superposition of identical one-dimensional dissipative solitons [39] in adjacent rows of particles propagated along any crystallographic axis. Solitons in each row are presented by density maxima localized at least at three particles and moving along the row. Solitons in adjacent rows form the wavefront which is oriented perpendicular to the direction of soliton propagation.

The propagation of flat solitons along any crystallographic axis is possible (Fig. 1b). The velocity of solitons in the lattice with parameters $b \sigma=5, \mu=1.41, v_{0}=0.14 \omega_{c} \sigma$ is equal to $\left|\vec{v}_{\text {sol }}\right| \approx 1.3$ in units $\omega_{c} \sigma$ in the coordinate system moving with the simulation cell. (Hence, the velocity of a soliton in a laboratory coordinate system is a sum $\vec{v}_{s o l}+\vec{v}_{l}$ where $\vec{v}_{l}$ is a simulation cell velocity. Its velocity depends on the number of solitons and orients arbitrarily relative to crystallographic axes.) The soliton velocity does not depend on initial conditions for the given values of $b \sigma, \mu$ and $v_{0}$. In the lattice considered, solitons are localized on an ordered set of particles. For this set at a given moment in time, the projection of the velocity vector on the direction of soliton propagation is directed oppositely to the projection of the average velocity of the ensemble, but approximately equal to it in magnitude. These projections cannot greatly exceed $v_{0}$ because nonlinear friction damps fast particles and accelerates slow ones.

In the simplest case the single soliton in a lattice with periodically boundary conditions is excited (Fig. 3a). However, in the general case the existence of a set of solitons is possible, as it is presented in Fig. 3b. The soliton-like excitations considered are metastable states. Their lifetime depends on soliton spatial distribution (as in a one-dimensional chain [39]), and on the degree of perturbation of the lattice. Perturbation can be caused by thermal fluctuations and, in computer experiments, by rounding errors in numerical simulation.

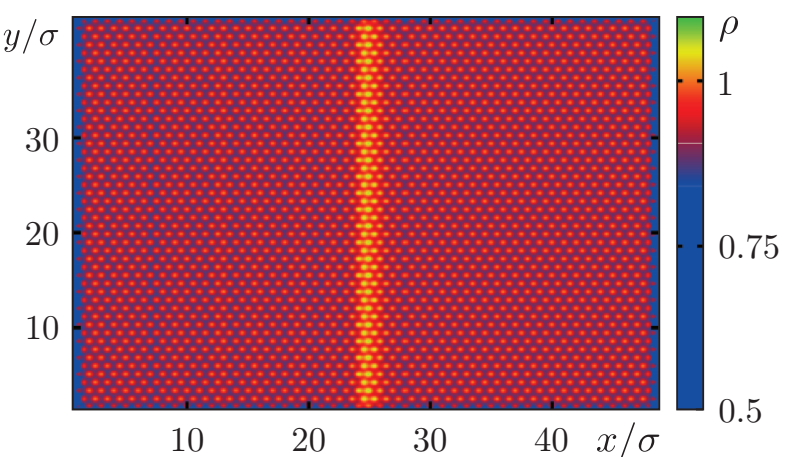

(a)

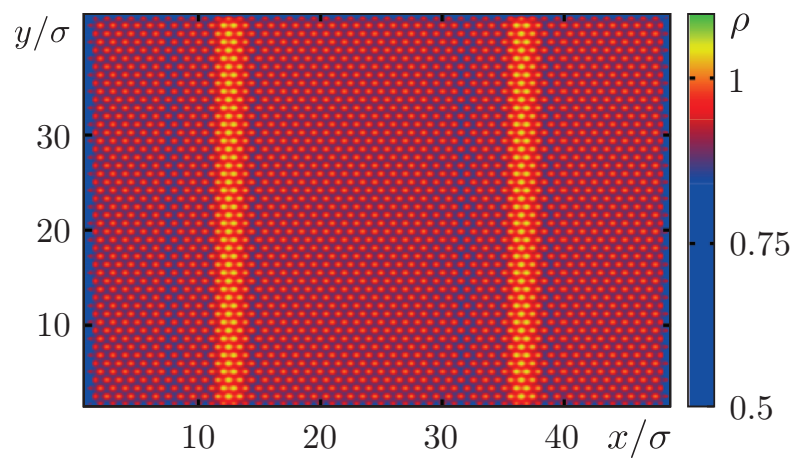

(b)

Fig. 3. Instantaneous spatial distributions of the particle density in the triangular lattice of $48 \times 48$ particles in the case of excitation of one (a) and two (b) solitons propagating along the $x$ axis. $b \sigma=5$, $\mu=1.41, v_{0}=0.14 \omega_{c} \sigma$. 
Collapse of a soliton is related to modulation instability. When particles get a little perturbation in the direction perpendicular to the direction of soliton propagation, this perturbation grows and the front of the soliton deforms and disappears.

Nevertheless, the plane solitons lifetimes values are large enough (of the order of $10^{3} / \omega_{c}$ ) and solitons can pass through the simulation cell hundreds of times. In this case the dynamical structure factor (DSF) [41] is very useful for quantitative analysis of dynamical processes.

In the simplest case for a flat soliton the dynamical structure factor $S(\omega, k)$ is governed by

$$
S(\omega, k)=\frac{1}{2 \pi} \int_{-\infty}^{\infty} e^{j \omega \tau}\left\langle\rho_{1 d}(k, \tau) \rho_{1 d}(-k, 0)\right\rangle d \tau,
$$

where the angular brackets show averaging for many realizations, $\omega$ and $k$ are the frequency and the wave number corresponding to the direction of propagation, and $\rho_{1 d}(x, \tau)$ and $\rho_{1 d}(k, \tau)$ are the density function and its spatial Fourier transform, respectively.

It is possible to choose one row along which the soliton propagates because all rows are identical. The selected row can be considered as a one-dimensional chain. Since the density of the point particles can be described by the delta function $\delta\left(x_{i}(\tau)\right)$, where $x_{i}(\tau)$ is the dimensionless particle coordinate in the row considered, we can introduce the density function of the onedimensional chain in the form

$$
\rho_{1 d}(x, \tau)=\sum_{i} \delta\left(x-x_{i}(\tau)\right)
$$

Then the spatial Fourier transform of the density function is

$$
\rho_{1 d}(k, \tau)=\sum_{i} e^{-j k x_{i}(\tau)},
$$

where $k$ is the wave number in units $1 / \sigma$.

The dependence of DSF on frequency is presented in Fig. 4. DSF has a single peak on the frequency corresponding to the period of the soliton passage through the simulation cell (Figs. 4a, 4b). In the simulation cell (which consists on $24 \times 24$ particles) this frequency is $0.36 \omega_{c}$ for a single soliton and $0.72 \omega_{c}$ for two solitons. Thus, DSF allows one to determine the time of the periodic passage of the soliton through the simulation cell, and then the velocity of the solitons.

Also, it is possible to determine the lifetime of a soliton by changing the time window for calculating SDF. For example, Fig. 4c demonstrates how the dynamical structure factor changes after soliton disintegration: the peak on the frequency of soliton passing is absent.

Moreover, the SDF calculations with different $k \sigma$ allow one to determine the total number of solitons, as shown in Fig. 4 d. The peak corresponding to $k \sigma=\pi / 6$ (i.e., two density maxima in a chain of 24 particles) is greater in the case of two solitons.

As noted above, one of the metastable states is the state with nonuniform distribution of solitons in the simulation cell (Fig. 5a).

Solitons move away from each other and finally they reach a steady-state configuration because they repel and tend to a configuration with the maximal distance between them. Correspondingly, the metastable state with nonuniform distribution of solitons transforms firstly to a stationary one with uniform distribution of solitons.

The duration of this process exponentially depends on parameter values and on the size of the simulation cell as in the one-dimensional chain of active particles [11]. This fact is

RUSSIAN JOURNAL OF NONLINEAR DYNAMICS, 2018, 14(2), 195-207 


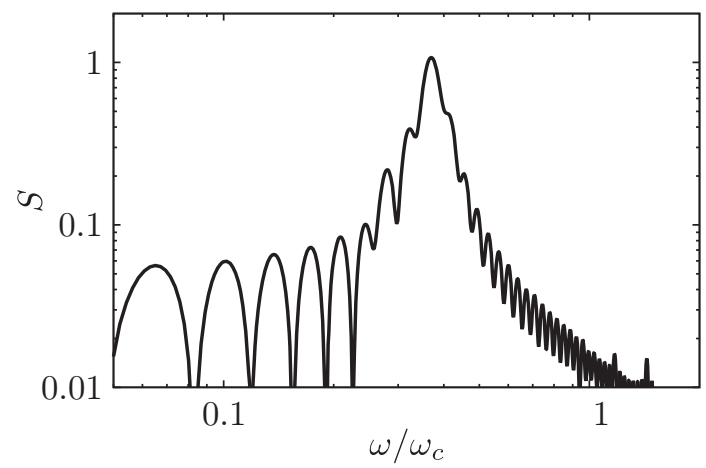

(a)

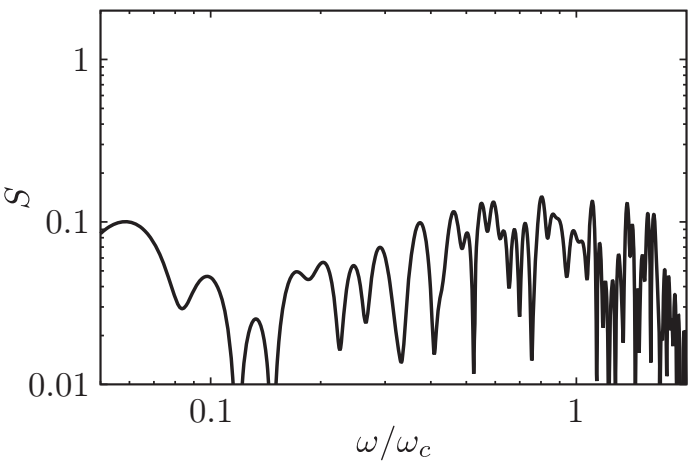

(c)

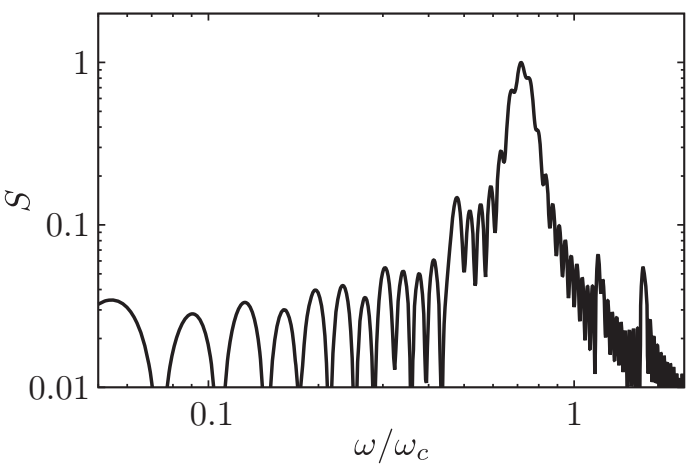

(b)

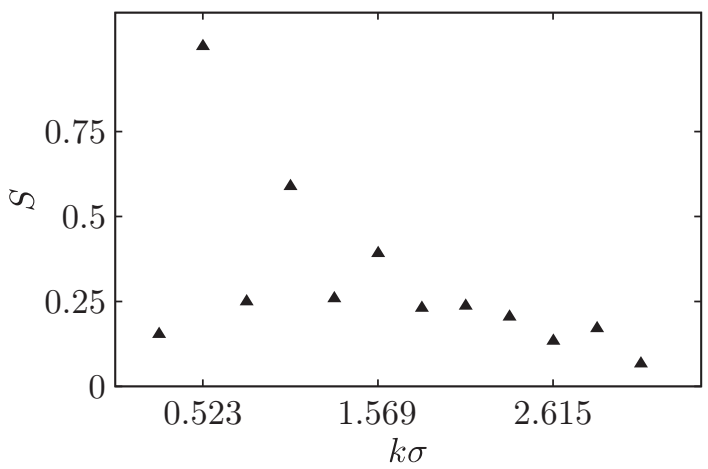

(d)

Fig. 4. Dynamical structure factor for a simulation cell consisting of $24 \times 24$ particles. (a) a single soliton with the wave number $k \sigma=\pi / 12$; (b) two solitons with the wave number $k \sigma=\pi / 6$; (c) two solitons, the time window for calculating the DSF is chosen after the moment of the soliton breaking, the wave number is $k \sigma=\pi / 6$; (d) the magnitude of the DSF peak for different wave numbers in the case of two solitons; the maximum corresponds to the wave number $k \sigma=\pi / 6$. $b \sigma=5, \mu=1.41, v_{0}=0.14 \omega_{c} \sigma$.

demonstrated in Fig. 6, which shows the dependence of transient time on the parameter $\mu$ for the case of two plane solitons initially separated by two rows of particles in the simulation cell of $12 \times 24$ particles.

As can be seen from the dependence, the duration of the transient process increases exponentially as the parameter $\mu$ increases. These results are qualitatively similar to those obtained earlier for the one-dimensional Morse-Rayleigh chains in [39].

Another type of metastable states is plane soliton-like waves with the wavefront oriented parallel to one of the crystallographic axes, which propagate perpendicularly to this axis (Fig. 5b). As the results of modeling demonstrate, such solitons have a smaller lifetime because of a greater transverse modulation instability.

Moreover, there exist plane solitons with finite width (Fig. 5c). They are metastable states with linear dependence between the lifetime (or maximum track length) and the width of a front. This dependence is presented in Fig. 7a. The initial conditions for such solitons are similar to those for the excitation of $M$-solitons or $M$-crowdions in the conservative lattice [30]. It is necessary to provide an initial momentum to $M$ particles of adjacent rows along a crystallographic axis. The selected particles will form the wavefront perpendicular to the direction of propagation. 


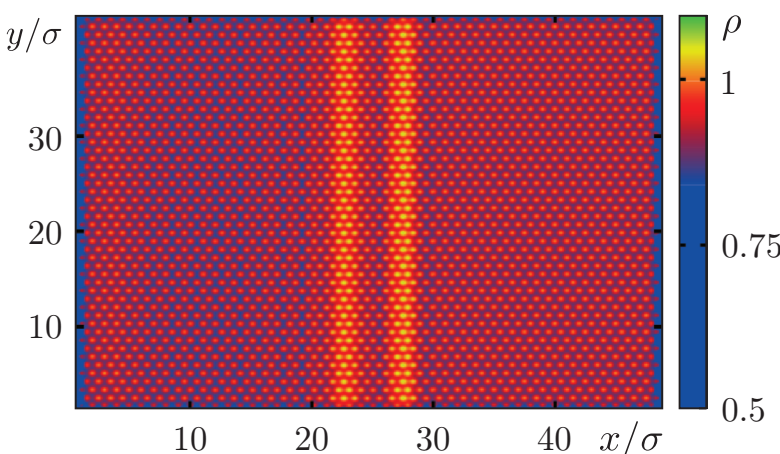

(a)

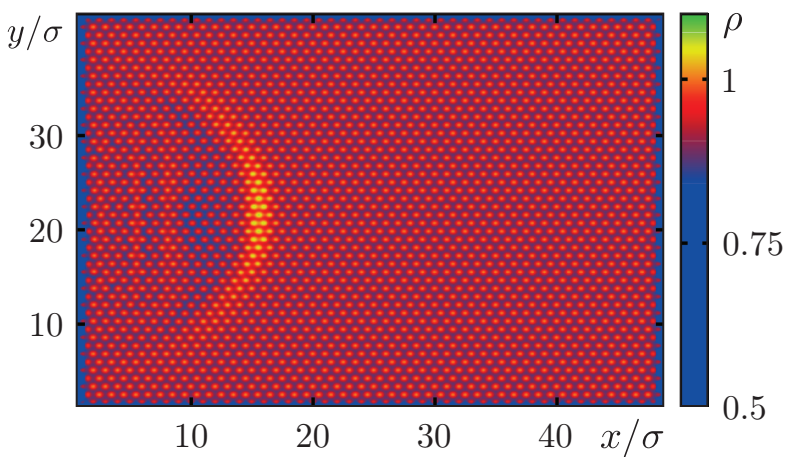

(c)

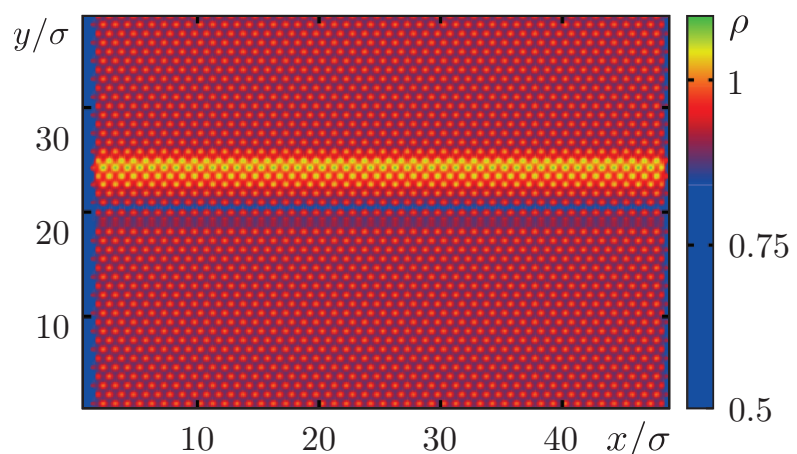

(b)

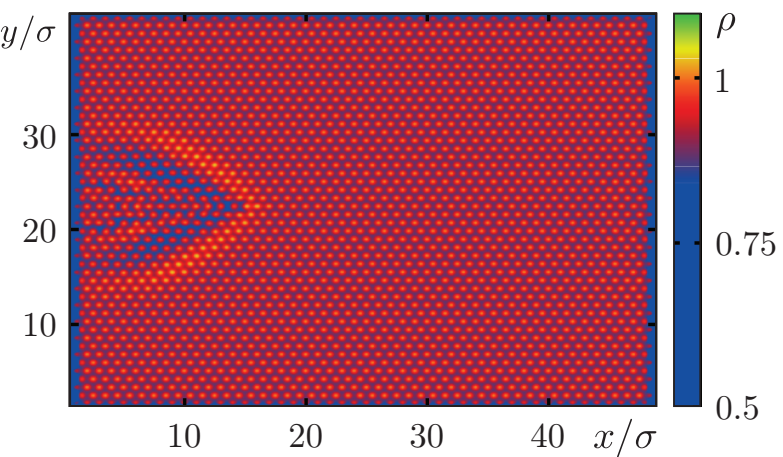

(d)

Fig. 5. Instantaneous spatial distribution of density for metastable states in the triangular lattice of $48 \times 48$ particles: (a) two plane solitons with an unequal distance between the density maxima; (b) a flat soliton with the wavefront parallel to the crystallographic axis (oriented along the $x$ axis), but the direction of propagation is oriented along the bisector of the angle between the two other axes (i.e., along the $y$ axis); (c) horseshoe-like shape soliton, initially localized on 15 particles; (d) quasi-one-dimensional soliton. The parameter values are $b \sigma=5, \mu=1.41, v_{0}=0.14 \omega_{c} \sigma$.

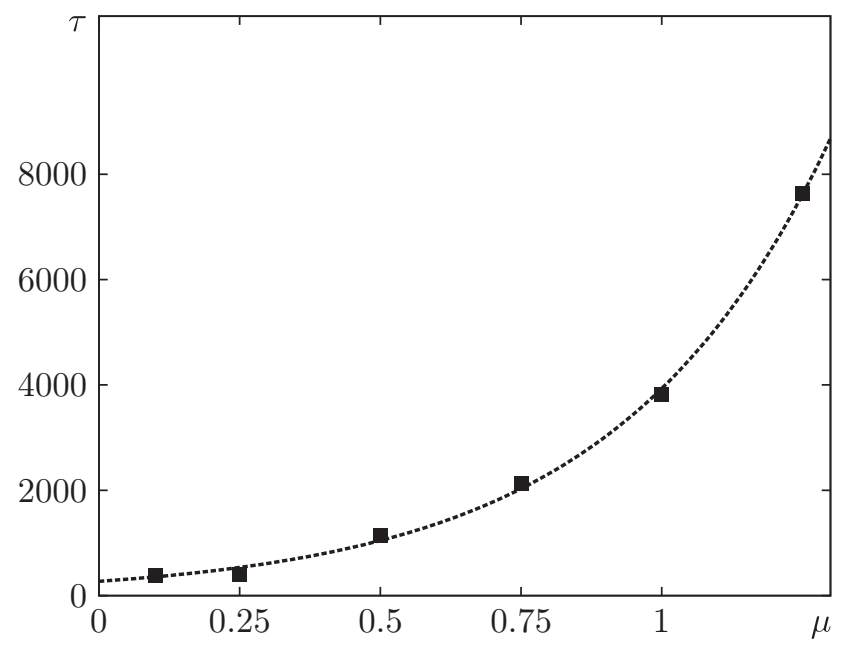

Fig. 6. Dependence of the time interval required to establish a uniform distribution of solitons on parameter $\mu$ (points) and its approximation by the function of type $f(x)=\alpha+\beta e^{\gamma x}$ (lines) in a lattice $12 \times 24$ particles. The parameter values are $b \sigma=5, v_{0}=0.14 \omega_{c} \sigma$. 
During the propagation of $M$-solitons the particles located at the front edges transmit a pulse not only in the direction of propagation of the soliton, but also in the perpendicular direction. As a result, the front obtains the horseshoe shape (Fig. 5c), it becomes narrow and eventually disappears. Correspondingly, the lattice goes to the translational mode.

In the limiting case the front width can be presented by only one particle; this excitation is called one-dimensional soliton (Fig. 5d). In the lattice considered this type of solitonlike excitation is a localized metastable state, which transforms into translational mode as in a conservative lattice $[25,27]$.

The lifetime and track length of a quasi-one-dimensional soliton depend on the potential rigidity and velocity of the initially perturbed particle $v_{i 0}$, as shown in Fig. $7 \mathrm{~b}$. There is some range of initial velocity in which the track length rapidly grows with increasing initial velocity. But with further increase in the velocity the track length does not grow and the dependence of the track length as a function of the initial energy reaches saturation. This behavior can be explained by damping of rapid particles under the action of negative friction.
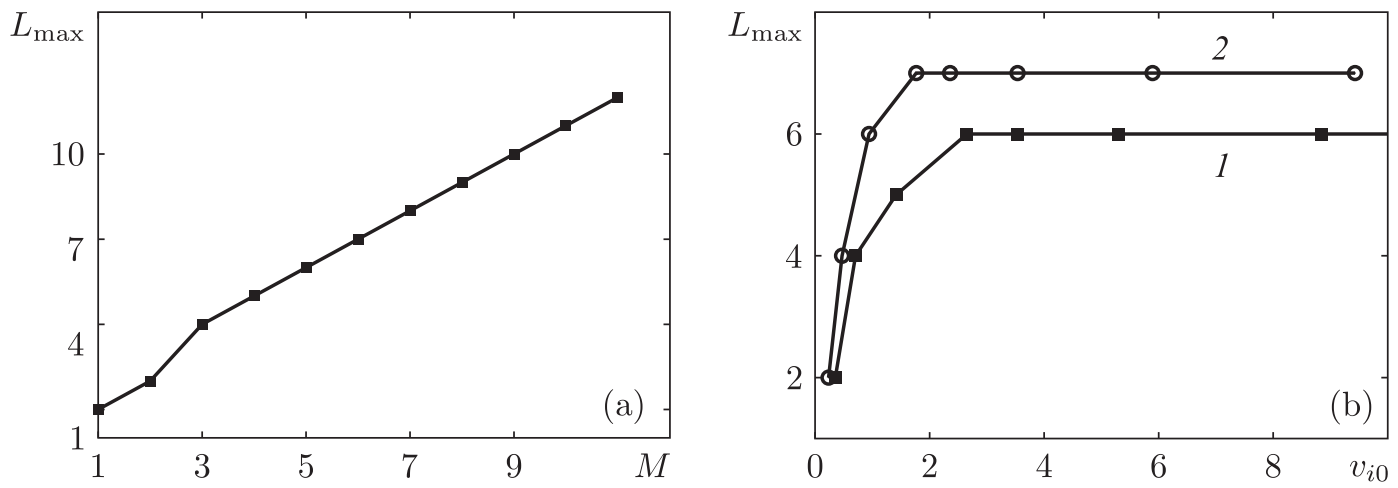

Fig. 7. (a) linear dependence of maximal track lengths on the width of the plane horseshoe-like shape soliton front. $b \sigma=5, \mu=1.41, v_{0}=0.14 \omega_{c} \sigma ;(\mathrm{b})$ dependences of the quasi-one-dimensional soliton maximal track length on the initial particle velocity for $b \sigma=4$ (line 1) and $b \sigma=6$ (line 2), $\mu=1.41$, $v_{0}=0.14 \omega_{c} \sigma$.

The chaotic (quasi-chaotic) transient process can be obtained when the system starts with a random initial distribution of the particles' velocities. This state is also metastable because incoherent oscillations of particles fade under the action of the nonlinear friction and the lattice tends to the translational mode. The lifetime of the chaotic regime depends on the number of particles in the ensemble and can be hundreds or thousands of dimensionless time units.

\section{Conclusion}

In the lattice of active particles with triangular symmetry, the set of existing stationary modes (attractors) is represented by the translational modes (lattice motion as a whole), both with a uniform distribution of particles in space and with topological defects.

However, it is possible to excite several types of metastable states that transform with time into a stationary state. Metastable states which transform to the translational mode are presented by single plane solitons and states with several uniformly distributed plane solitons (lifetimes of the order of $10^{3} / \omega_{c}$ ), horseshoe $M$-solitons (lifetimes of the order of $10^{2} / \omega_{c}$ ), and quasi-one-dimensional solitons. All these metastable solitons propagate along the crystallographic axes. 
There are also plane solitons propagating in the directions perpendicular to the crystallographic axes. Such solitons are subject to the phenomena of modulation instability, and the characteristic lifetime of these states is of the order of $10 / \omega_{c}$.

Chaotic oscillations also can be attributed to metastable states; this regime can be obtained in the case of stochastic initial conditions.

Sets of nonuniformly distributed plane solitons are metastable states and, depending on the initial conditions and the size of the simulation cell, they can transform either directly to the translational mode or firstly to the stationary state with uniform distribution of solitons and then to the translational mode.

The formation of any ordered state in the lattice considered requires special initial conditions. The lifetimes and the track lengths of different metastable states depend mainly on the initial conditions.

\section{References}

[1] Romanczuk, P., Bar, M., Ebeling, W., Lindner, B., and Schimansky-Geier, L., Active Brownian Particles: From Individual to Collective Stochastic Dynamics, Eur. Phys. J. Special Topics, 2012, vol. 202, no. 1, pp. 1-162.

[2] Saintillan, D. and Shelley, M. J., Instabilities and Pattern Formation in Active Particle Suspensions: Kinetic Theory and Continuum Simulations, Phys. Rev. Lett., 2008, vol.100, no. 17, 178103, 4 pp.

[3] Farrell, F.D.C., Marchetti, M.C., Marenduzzo, D., and Tailleur, J., Pattern Formation in SelfPropelled Particles with Density-Dependent Motility, Phys. Rev. Lett., 2012, vol. 108, no. 24, 248101, 5 pp.

[4] Schweitzer, F., Brownian Agents and Active Particles: Collective Dynamics in the Natural and Social Sciences, Berlin: Springer, 2003.

[5] Bechinger, C., Di Leonardo, R., Löwen, H., Reichhardt, Ch., Volpe, Giorgio, and Volpe, Giovanni, Active Particles in Complex and Crowded Environments, Rev. Modern Phys., 2016, vol. 88, no. 4, 045006, 50 pp.

[6] Marchetti, M., Fily, Ya., Henkes, S., Patch, A., and Yllanes, D., Minimal Model of Active Colloids Highlights the Role of Mechanical Interactions in Controlling the Emergent Behavior of Active Matter, Curr. Opin. Colloid Interface Sci., 2016, vol.21, pp. 34-43.

[7] Balboa Usabiaga, F., Kallemov, B., Delmotte, B., Bhalla, A.P.S., Griffith, B. E., and Donev, A., Hydrodynamics of Suspensions of Passive and Active Rigid Particles: A Rigid Multiblob Approach, Commun. Appl. Math. Comput. Sci., 2016, vol.11, no. 2, pp. 217-296.

[8] Alarcón, F. and Pagonabarraga, I., Spontaneous Aggregation and Global Polar Ordering in Squirmer Suspensions, J. Mol. Liq., 2013, vol. 185, pp. 56-61.

[9] Romensky, M., Scholz, D., and Lobaskin, V., Hysteretic Dynamics of Active Particles in a Periodic Orienting Field, J. R. Soc. Interface, 2015, vol. 12, no. 108, 20150015.

[10] Wensink, H.H., Kantsler, V., Goldstein, R.E., and Dunkel, J., Controlling Active Self-Assembly through Broken Particle-Shape Symmetry, Phys. Rev. E, 2014, vol. 89, no. 1, 010302(R), 5 pp.

[11] Sergeev, K.S., Vadivasova, T.E., and Chetverikov, A. P., Noise-Induced Transition in a Small Ensemble of Active Brownian Particles, Tech. Phys. Lett., 2014, vol.40, no.11, pp.976-979; see also: Pis'ma Zh. Tekh. Fiz., 2014, vol.40, no. 21, pp.88-96.

[12] Suchkov, S. V., Sukhorukov, A. A., Huang, J., Dmitriev, S. V., Lee, C., and Kivshar, Yu. S., Nonlinear Switching and Solitons in PT-Symmetric Photonic Systems, Laser Photonics Rev., 2016, vol.10, no. 2, pp. 177-213.

[13] Barashenkov, I. V., Suchkov, S. V., Sukhorukov, A. A., Dmitriev, S. V., and Kivshar, Yu.S., Breathers in PT-Symmetric Optical Couplers, Phys. Rev. A, 2012, vol. 86, no. 5, 053809, 12 pp.

RUSSIAN JOURNAL OF NONLINEAR DYNAMICS, 2018, 14(2), 195-207 
[14] Saadatmand, D., Borisov, D. I., Kevrekidis, P. G., Zhou, K., and Dmitriev, S. V., Resonant Interaction of $\phi^{4}$ Kink with PT-Symmetric Perturbation with Spatially Periodic Gain/Loss Coefficient, Commun. Nonlinear Sci. Numer. Simul., 2018, vol.56, pp. 62-76.

[15] Borisov, D. I. and Dmitriev, S. V., On the Spectral Stability of Kinks in 2D Klein-Gordon Model with Parity-Time-Symmetric Perturbation, Stud. Appl. Math., 2017, vol. 138, no. 3, pp. 317-342.

[16] Saadatmand, D., Dmitriev, S. V., Borisov, D. I., Kevrekidis, P. G., Fatykhov, M. A., and Javidan K., Effect of the $\phi^{4}$ Kink's Internal Mode at Scattering on a PT-Symmetric Defect, JETP Lett., 2015, vol.101, no.7, pp.497-502; see also: Pis'ma v Zh. Èksper. Teoret. Fiz., 2015, vol.101, no. 7 , pp. 550-555.

[17] Saadatmand, D., Dmitriev, S. V., Borisov, D. I., Kevrekidis, P. G., Fatykhov, M. A., and Javidan K., Kink Scattering from a Parity-Time-Symmetric Defect in the $\phi^{4}$ Model, Commun. Nonlinear Sci. Numer. Simul., 2015, vol. 29, nos. 1-3, pp. 267-282.

[18] Saadatmand, D., Dmitriev, S. V., Borisov, D. I., and Kevrekidis, P. G., Interaction of sine-Gordon Kinks and Breathers with a Parity-Time-Symmetric Defect, Phys. Rev. E, 2014, vol.90, no.5, 052902, 10 pp.

[19] Takatori, S. C. and Brady, J.F., A Theory for the Phase Behavior of Mixtures of Active Particles, Soft Matter, 2015, vol.11, no. 40, pp.7920-7931.

[20] Mallory, S. A., Valeriani, C., and Cacciuto, A., Anomalous Dynamics of an Elastic Membrane in an Active Fluid, Phys. Rev. E, 2015, vol. 92, no. 1, 012314, 6 pp.

[21] Huepe, C., Ferrante, E., Wenseleers, T., and Turgut, A. E., Scale-Free Correlations in Flocking Systems with Position-Based Interactions, J. Stat. Phys., 2015, vol. 158, no. 3, pp. 549-562.

[22] Ferrante, E., Turgut, A. E., Dorigo, M., and Huepe, C., Collective Motion Dynamics of Active Solids and Active Crystals, New J. Phys., 2013, vol. 15, 095011, 20 pp.

[23] Jones, J.E., On the Determination of Molecular Fields: 2. From the Equation of State of a Gas, Proc. Roy. Soc. London Ser. A, 1924, vol. 106, no. 738, pp. 463-477.

[24] Morse, Ph. M., Diatomic Molecules According to the Wave Mechanics: 2. Vibrational Levels, Phys. Rev., 1929, vol. 34, no. 1, pp. 57-64.

[25] Chetverikov, A.P., Ebeling, W., and Velarde, M. G., Localized Nonlinear, Soliton-Like Waves in Two-Dimensional Anharmonic Lattices, Wave Motion, 2011, vol.48, no. 8, pp. 753-760.

[26] Ikeda, K., Doi, Y., Feng, B.-F., and Kawahara, T., Chaotic Breathers of Two Types in a TwoDimensional Morse Lattice with an On-Site Harmonic Potential, Phys. D, 2007, vol. 225, no. 2, pp. $184-196$.

[27] Chetverikov, A.P., Ebeling, W., and Velarde, M. G., Soliton-Like Excitations and Solectrons in Two-Dimensional Nonlinear Lattices, Eur. Phys. J. B, 2011, vol. 80, no. 2, pp. 137-145.

[28] Chetverikov, A. P., Ebeling, W., and Velarde, M. G., Properties of Nano-Scale Soliton-Like Excitations in Two-Dimensional Lattice Layers, Phys. D, 2011, vol. 240, no. 24, pp. 1954-1959.

[29] Korznikova, E. A., Fomin, S. Yu., Soboleva, E. G., and Dmitriev, S. V., Highly Symmetric Discrete Breather in a Two-Dimensional Morse Crystal, JETP Lett., 2016, vol. 103, no.4, pp. 277-281; see also: Pis'ma v Zh. Èksper. Teoret. Fiz., 2016, vol. 103, no. 4, pp. 303-308.

[30] Chetverikov, A. P., Shepelev, I. A., Korznikova, E. A., Kistanov, A. A., Dmitriev, S. V., and Velarde, M. G., Breathing Subsonic Crowdion in Morse Lattices, Comput. Condens. Matter, 2017, vol. 13, pp. 59-64.

[31] Dmitriev, S. V., Medvedev, N. N., Chetverikov, A. P., Zhou, K., and Velarde, M. G., Highly Enhanced Transport by Supersonic N-Crowdions, Phys. Status Solidi RRL, 2017, vol. 11, no. 12, 1700298, 5 pp.

[32] Dmitriev, S. V., Korznikova, E.A., and Chetverikov, A.P., Supersonic N-Crowdions in a TwoDimensional Morse Crystal, JETP, 2018, vol.126, no. 3, pp.347-352; see also: Zh. Èksper. Teoret. Fiz., 2018, vol. 153, no. 3, pp. 417-423.

[33] Korznikova, E. A., Bachurin, D. V., Fomin, S. Yu., Chetverikov, A. P., and Dmitriev, S. V., Instability of Vibrational Modes in Hexagonal Lattice, Eur. Phys. J. B, 2017, vol. 90, no. 2, 23, 8 pp. 
[34] Korznikova, E. A., Kistanov, A. A., Sergeev, K.S., Shepelev, D. A., Davletshin, A. R., Bokii, D. I., and Dmitriev, S. V., The Reason for Existence of Discrete Breathers in 2D and 3D Morse Crystals, Letters on Materials, 2016, vol.6, no.3, pp. 221-226 (Russian).

[35] Dmitriev, S. V., Korznikova, E. A., Baimova, Yu. A., and Velarde, M. G., Discrete Breathers in Crystals, Physics-Uspekhi, 2016, vol.59, no.5, pp.446-461; see also: Uspekhi Fiz. Nauk, 2016, vol. 186, no. 5, pp. 471-488.

[36] Makarov, V.A., del Rio, E., Ebeling, W., Velarde, M. G., Dissipative Toda-Rayleigh Lattice and Its Oscillatory Modes, Phys. Rev. E, 2001, vol. 64, no. 3, 036601, 14 pp.

[37] Ebeling, W., Landa, P.S., and Ushakov, V. G., Self-Oscillations in Ring Toda Chains with Negative Friction, Phys. Rev. E, 2001, vol. 63, no. 4, 046601, 8 pp.

[38] del Rio, E., Makarov, V.A., Velarde, M. G., and Ebeling, W., Mode Transitions and Wave Propagation in a Driven-Dissipative Toda-Rayleigh Ring, Phys. Rev. E, 2003, vol.67, no. 5, 056208, 9 pp.

[39] Sergeev, K.S. and Chetverikov, A.P., Metastable States in the Morse-Rayleigh Chain, Nelin. Dinam., 2016, vol. 12, no. 3, pp. 341-353 (Russian).

[40] Velarde, M. G., Solitons as Dissipative Structures, Int. J. Quant. Chem., 2004, vol.98, no.2, pp. 272-280.

[41] Chetverikov, A.P., Ebeling, W., Velarde, M. G., Solitons and Clusters in One-Dimensional Ensembles of Interacting Brownian Particles, Izv. SGU. Novaya Seriya. Fizika, 2006, vol.6, nos. 1-2, pp. 28-41 (Russian). 